\title{
Further characterisation of a spontaneously occurring antibasement membrane antibody
}

\author{
ARTHUR J. AMMANN AND JOSEPH R. GOODMAN \\ From the Department of Pediatrics, Immunology Section, University of California, San Francisco, and \\ Veterans Administration, San Francisco, California, USA
}

SUMMARY A spontaneously occurring antibasement membrane antibody has been further characterised according to antigenic specificity, immunoglobulin class, and tissue localisation using immunofluorescent and immunoelectron microscopic techniques. The autoantibody reacted with the basement membrane of kidney tubules, Bowman's capsule, and the epithelial portion of ileum but not with the basement membrane of skin, cornea, glomerulus, or oesophagus. It also reacted with bile canaliculi, sarcolemmal sheath, and salivary duct. On electron microscopy the antibody was distributed along the basement membrane of Bowman's capsule and renal tubules. Some reactivity against collagen was observed. Antibody activity was found in both IgG and IgM fractions. In immunodeficiency disorders, the autoantibody was found only in patients with selective $\operatorname{IgA}$ deficiency.

A spontaneously occurring autoantibody reacting with selected membranes and distinct from other basement membranes was described by us in 1971 (Ammann and Hong, 1971a). This antibody was found in patients with coeliac disease and $\operatorname{IgA}$ deficiency. Seah et al. (1971) had previously described an autoantibody with similar tissue localisation, which they termed reticulin antibody and which they found primarily in patients with coeliac disease and dermatitis herpetiformis. Subsequent studies by Alp and Wright (1971) and von Essen et al. (1972) confirmed both the tissue localisation of the autoantibody and the occurrence in coeliac disease. Rizzetto and Doniach (1973) reported on the occurrence of reticulin antibodies in an extensive evaluation of 3628 sera from patients with a wide variety of disorders. They defined the tissue localisation of the autoantibody, the specificity for certain disease states, and the immunoglobulin class distribution. They further suggested that five types of autoantibody could be demonstrated and termed these: reticulin $\mathbf{R}_{1}$, reticulin $\mathbf{R}_{\mathbf{2}}$, Kupffer cell, sinusoidal adherent cell cytoplasmic, and reticulin sinusoidal Rs.

In this report we describe detailed studies on the tissue localisation and distribution of antibody

Received for publication 14 July 1977

Amended version accepted 12 December 1977 reactive with basement membrane using immunofluorescent and immunoelectron microscopic techniques. This autoantibody appears to be identical with that described by Seah et al. (1971) and the reticulin $R_{1}$ antibody described by Rizzetto and Doniach (1973).

\section{Material and methods}

Blood samples were obtained from patients, immediately separated, and stored frozen at $-20^{\circ} \mathrm{C}$. These samples were used to perform indirect immunofluorescence at 1-10 serum dilutions on various tissues obtained from rat and human sources, as previously described (Ammann and Hong, 1971b).

Electron microscopy was performed as follows: Normal rat kidney was cut and placed in ice-cold $1 \%$ glutaraldehyde in $0.1 \mathrm{M}$ cacodylate buffer for 45 minutes. Blocks were washed in buffer and $35 \mu$ slices were cut on a tissue chopper. This was followed by incubation with either the patient's purified IgG or normal human serum for 45 minutes at room temperature and the sections were washed three times with $0 \cdot 1 \mathrm{M}$ cacodylate buffer. These, plus a third control preparation not incubated with serum, were all incubated in ferritinlabelled goat anti-human IgG for 30 minutes, washed three times with $0.1 \mathrm{M}$ cacodylate buffer, fixed in 
buffered $1 \%$ glutaraldehyde, post-fixed in $1 \%$ osmium, and prepared for electron microscopy.

Collagen was prepared from the skin of newborn infants and was supplied to us by Rothbard and Watson (1972). This preparation was used for absorption of antibody in a concentration of $1 \mathrm{mg} / \mathrm{ml}$ with varying amounts of serum.

The autoantibody was separated into IgG and IgM fractions, using sodium sulphate precipitation and Sephadex G200 column chromatography. The patient from whom this autoantibody was obtained lacked serum $\operatorname{IgA}$.

\section{Results}

Separation of the serum into IgG and IgM fractions and subsequent indirect immunofluorescence with anti-IgG and anti-IgM antibody indicated that autoantibody activity was present in both immunoglobulin fractions. No autoantibody activity was present in IgA as the patient lacked serum IgA.

After absorption of the autoantibody with highly purified collagen, no appreciable change in the distribution or intensity of autoantibody reactivity could be demonstrated by immunofluorescent techniques. Table 1 summarises the distribution of reactivity of the autoantibody against various tissues. Figure 1 demonstrates a typical pattern on rat renal tubules.
Table 1 Tissue reactivity of antibasement membrane activity

\begin{tabular}{|c|c|}
\hline Structure & $\begin{array}{l}\text { Anti-IgG } \\
\text { fluorescent antibody } \\
\text { reactivity }\end{array}$ \\
\hline Bowman's capsule & + \\
\hline Glomerular basement membrane & - \\
\hline Kidney tubule basement membrane & + \\
\hline Liver portal tracts & + \\
\hline Sarcolemmal sheath & + \\
\hline Epithelial cell basement membrane (ileum) & + \\
\hline Oesophagus basement membrane & - \\
\hline Salivary duct (striated duct) basement & \\
\hline membrane of serous cells & + \\
\hline Cornea basement membrane & - \\
\hline
\end{tabular}

Electron microscopy using ferritin-labelled anti $\dot{\omega}$ human gamma chain indicated that the autoantibody $N$ reacted in the same distribution on renal basemente membranes as was found using the immuno-fluorescent method. The ferritin-labelled anti-human? IgG was seen along many tubular basement mem $\vec{\imath}$ branes and in some endothelial cells of capillaries in tubular areas (Fig. 2). There was no ferritin anti= human IgG along glomerular basement membrane of glomerular capillary endothelium. In addition, if was present in the rat plasma in both tubule an ${ }^{\circ}$ glomerular capillary lumens, indicating that thes reagent penetrated to these areas and cross-reactedo with rat IgG. In certain areas activity agains collagen fibres was demonstrated (Fig. 3).

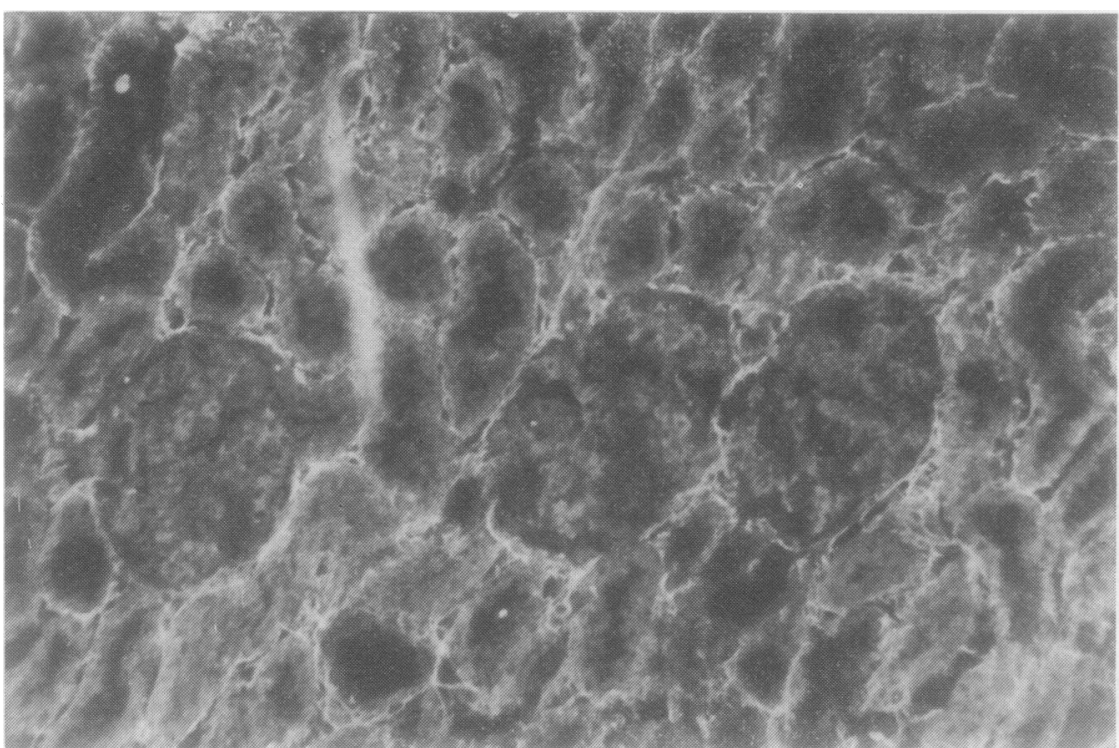

Fig. 1 Fluorescent photomicrograph of isolated IgG on rat kidney section. Positive staining of basement membrane of Bowman's capsule and tubules is seen. $\times 40$ 


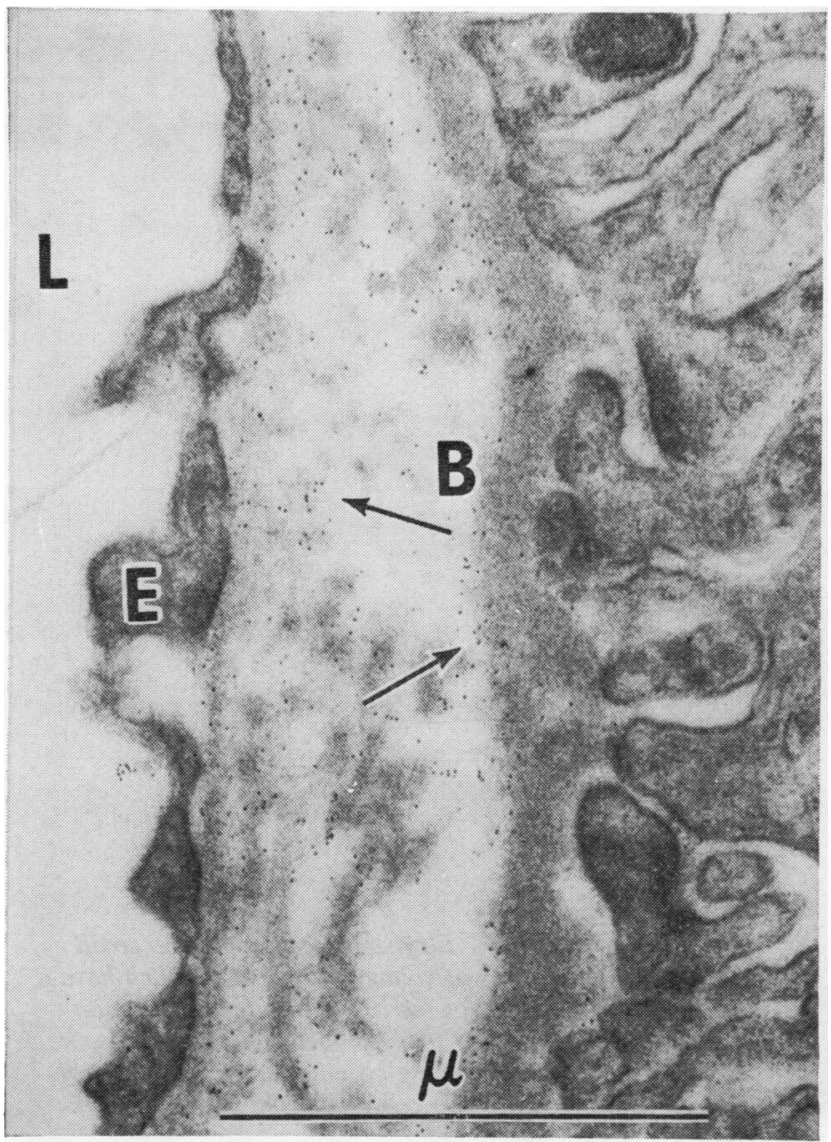

Table 2 summarises the distribution of antibasement membrane antibody in various clinical immunodeficiency disorders. It was not found in $\mathbf{4 0}$ normal adults and children.

\section{Discussion}

Spontaneously occurring antibasement membrane antibody has been described with varying histological distributions. In Goodpasture's syndrome, an autoantibody is found by immunofluorescence which reacts with the basement membrane of the renal glomerulus and lung (Lerner et al., 1967; McPhaul and Dixon, 1969). Autoantibody reacting with the basement membrane of skin and oesophagus has been found in pemphigoid (Chorzelski et al., 1966; Beutner et al., 1967). The autoantibody described in this report reacts with the basement membrane of Bowman's capsule and kidney tubules (Fig. 1) but not with the glomerulus or basement membrane of oesophagus or cornea. Reactivity was also observed with basement membrane of the epithelial cells of villi taken from the ileum, sarcolemmal sheath of muscle, and the portal tracts of the liver. In some sections reactivity with collagen fibres could be seen (Fig. 3). From these studies it cannot be determined if the autoantibodies are reacting with shared antigenic components or if two distinct autoantibodies are present. Attempts to absorb the autoantibody with collagen did not reduce antibasement membrane activity.

The importance of these studies is in the observation that both basement membrane and collagen reactivity were observed on electron microscopic evaluation whereas dual reactivity could not be distinguished by immunofluorescent studies. It is likely, therefore, that the antibasement membrane antibody described by us (Ammann and Hong, 1971b), the antireticulin antibody described by Seah et al. (1971), and the $\mathrm{R}_{1}$ antibody described by Rizzetto and Doniach (1973) have similar, if not identical, antigenic components, namely, basement membrane and collagen.

The studies of Seah et al. (1971) and the detailed 


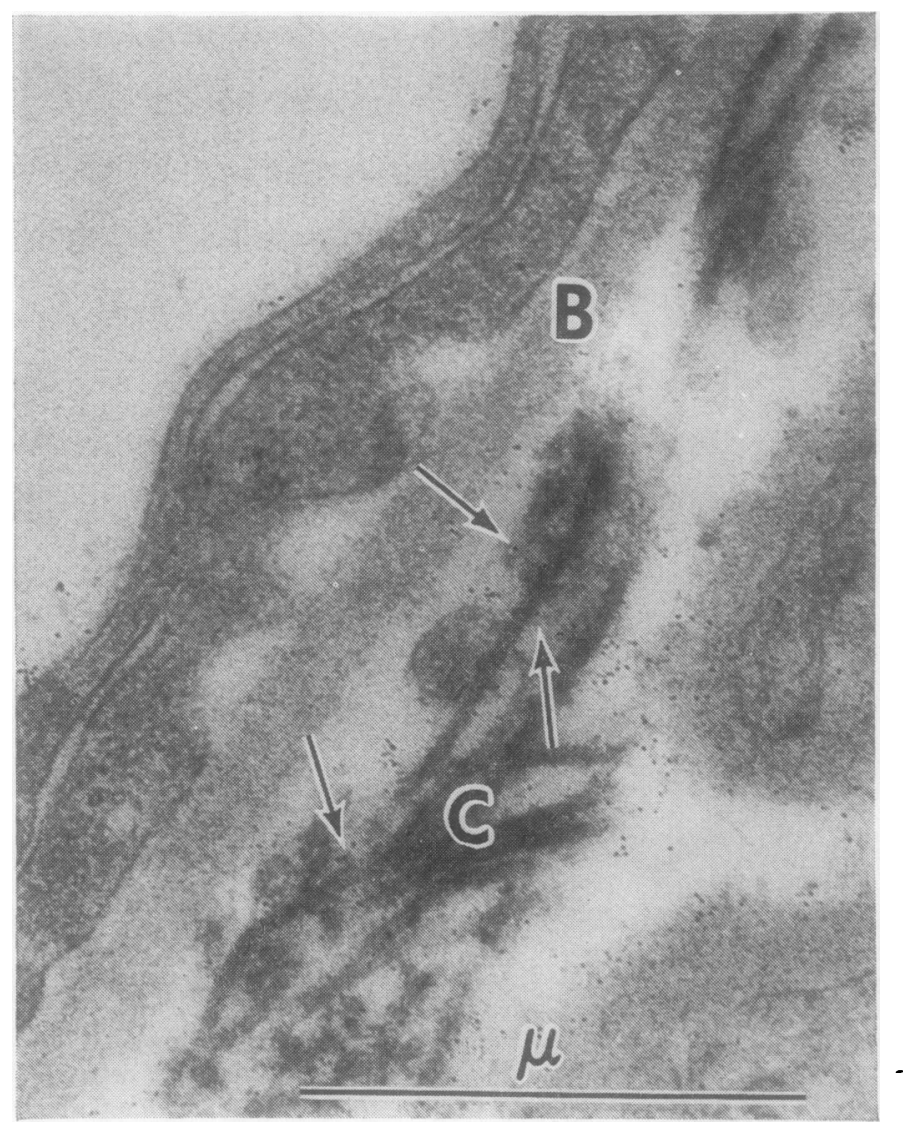

Fig. 3 Electron micrograph of a small cluster of collagen fibres $(C)$, which have a scatter of ferritin molecules (arrows) on and near their surface. There is also some ferritin in the basement membrane $(B)$ of this tubule cell. The scale is one micron.
Table 2 Antibasement membrane antibodies in immunodeficiency disorders

\begin{tabular}{lcl}
\hline Disorder & No. tested & No. positive \\
\hline $\begin{array}{l}\text { Coeliac disease with selective IgA } \\
\text { deficiency }\end{array}$ & 3 & 3 \\
Selective IgA deficiency without & 40 & 1 \\
$\quad$ coeliac disease & 12 & 0 \\
Hypogammaglobulinaemia & 6 & 0 \\
Thymic hypoplasia & 10 & 0 \\
Chronic granulomatous disease & 5 & 0 \\
Severe combined immunodeficiency & 40 & 0 \\
Normals (2 to 40 years) & & \\
\hline
\end{tabular}

investigations of Rizzetto and Doniach (1973) are in agreement as to the tissue localisation of antibody reactivity. A nodular fluorescence is observed surrounding the portal tracts of the liver with discrete wave-like bands extending into the sinusoidal walls, a honeycomb appearance on smooth or striated muscle, staining of Bowman's capsule, and reactivity in the peritubular area of the kidney. We have extended the number of tissues examined and find reactivity along the basement membrane of epithelial cells of ileum and basement membrane of salivaryo duct (striated duct) serous cells (Table 1). Nō reactivity was observed against basement membranes of cornea, glomerulus or oesophagus, indicating thato these basement membranes are distinct antigenically from those of Bowman's capsule, epithelial cells of? gastrointestinal tract and kidney tubule, and thes portal tract of the liver. It is of interest that the distinct distribution of antibasement membrane antibody detected in Goodpasture's syndrome, pemphigoid, and in coeliac disease corresponds to distincto differences in biochemical composition, as described by Kefalides (1969).

Several experimental studies suggest that antiro bodies to collagen and basement membrane have similar patterns of distribution by immunofluorescent $t^{?}$ techniques. Nagasawa and Shibata (1969) used human glomerular basement membrane as antigen? and studied the tissue distribution of antibody in rato tissue. Antibody activity was demonstrated against侣 the basement membrane of Bowman's capsule, renalo tubular glomerulus, sarcolemmal muscle sheath, and 
liver sinusoids. However, using histochemical techniques, Velican and Velican (1970) demonstrated structural heterogeneity between glomerular, capsular, and tubular basement membranes. Using antibody to human infant skin collagen, Rothbard and Watson (1972) demonstrated reactivity by immunofluorescent methods in a pattern of distribution similar to that obtained using antibasement membrane antibody. These studies emphasise the difficulty in distinguishing antibasement membrane activity from anti-collagen activity by immunofluorescent methods alone.

Rizzetto and Doniach (1973) stressed that connective tissue elements are a mixture of amorphous proteins and mucopolysaccharides in which there are fibrous proteins related to collagen. They suggested that electron microscopic studies would be necessary to see if the antigens detected by spontaneously occurring autoantibody are fibrillary or belong to amorphous proteins and mucopolysaccharides. Our studies clearly demonstrate both basement membrane and collagen reactivity. The term 'reticulin' antibody (Seah et al., 1971) or ' $R_{1}$ ' antibody (Rizzetto and Doniach, 1973) would, therefore, appear to be appropriate general terms when this autoantibody is detected by immunofluorescent methods. The antibody activity may be further defined as against basement membrane and/or collagen by electron microscopic techniques.

In clinical disorders, the autoantibody has been found to be primarily associated with coeliac disease. Although we originally described the autoantibody in patients with selective IgA deficiency coeliac disease (Ammann and Hong, 1971b), Seah et al. (1971) and Alp and Wright (1971) found the autoantibody to be present in patients with coeliac disease, the majority of whom had normal IgA values. In a study of 3628 sera from patients with a variety of diseases, Rizzetto and Doniach (1973) found antibody to reticulin to be most frequently present in dermatitis herpetiformis, coeliac disease, chronic heroin addicts, Sjögren's syndrome, myasthenia gravis, vitiligo, alopecio, and rheumatoid arthritis. However, they did not distinguish between the various subtypes of antibody to reticulin present in the various diseases. Spontaneously occurring antitubular basement membrane antibody has been described after renal transplantation (Wilson et al., 1974) and in methicillin-induced interstitial nephritis (Baldwin et al., 1975).

We studied the sera of 76 patients with immunodeficiency to determine the relationship between antibody against reticulin and specific immunodeficiency disorders. Although the number of patients in some categories was small, only one patient who did not have coeliac disease was found to be positive. This patient had selective IgA deficiency and oedema of unknown aetiology. Thus, the autoantibody appears to be confined to immunodeficient patients with selective IgA deficiency.

This study was supported by John A. Hartford Foundation, Inc., and the Division of Research Resources, National Institutes of Health 5 MO1RR00079-12.

\section{References}

Alp, M. H., and Wright, R. (1971). Autoantibodies to reticulin in patients with idiopathic steatorrhoea, coeliac disease, and Crohn's disease, and their relation to immunoglobulins and dietary antibodies. Lancet, 2, 682-685.

Ammann, A. J., and Hong, R. (1971a). Unique antibody to basement membrane in patients with selective IgA deficiency and coeliac disease. Lancet, 1, 1264-1266.

Ammann, A. J., and Hong, R. (1971b). Selective IgA deficiency. Presentation of 30 cases and a review of the literature. Medicine, 50, 223-236.

Baldwin, D. S., Levine, B. B., McCluskey, R. T., and Gallo, G. R. (1975). Renal failure and interstitial nephritis due to penicillin and methicillin. New England Journal of Medicine, 279, 1245-1252.

Beutner, E. H., Rhodes, E. L., and Holborow, E. J. (1967). Autoimmunity in chronic bullous diseases. Immunofluorescent demonstration of antibodies to skin in sera of patients with pemphigus, bullous pemphigoid and in other human sera. Clinical and Experimental Immunology, 2, 141-151.

Chorzelski, T. P., Von Weiss, J. F., and Lever, W. F. (1966). Clinical significance of autoantibodies in pemphigus. Archives of Dermatology, 93, 570-576.

von Essen, R., Savilahti, E., and Pelkonen, P. (1972). Reticulin antibody in children with malabsorption. Lancet, 2, 1157-1159.

Kefalides, N. A. (1969). The chemistry and structure of basement membranes. Arthritis and Rheumatism, 12, 427-443.

Lerner, R. A., Glassock, R. J., and Dixon, F. J. (1967). The role of anti-glomerular basement membrane antibody in the pathogenesis of human glomerulonephritis. Journal of Experimental Medicine, 126, 989-1004.

McPhaul, J. J., Jr., and Dixon, F. J. (1969). The presence of anti-glomerular basement membrane antibodies in peripheral blood. Journal of Immunology, 103, 11681175 .

Nagasawa, T., and Shibata, S. (1969). Immunofluorescence studies on the anatomic distribution of the soluble antigen responsible for the production of nephrotoxic serum nephritis. Journal of Immunology, 103, 736-740.

Rizzetto, M., and Doniach, D. (1973). Types of 'reticulin' antibodies detected in human sera by immunofluorescence. Journal of Clinical Pathology, 26, 841-851. 
Rothbard, S., and Watson, R. F. (1972). Demonstration of collagen in human tissues by immunofluorescence. Laboratory Investigation, 27, 76-84.

Seah, P. P., Fry, L., Hoffbrand, A. V., and Holborow, E. J. (1971). Tissue antibodies in dermatitis herpetiformis and adult coeliac disease. Lancet, 1, 834-836.

Velican, D., and Velican, C. (1970). Structural heterogeneity of kidney basement membranes. Nature, 226, 1259-1261.
Wilson, C. B., Lehman, D. H., McCoy, R. C., Gunnells, J. C., Jr., and Stickel, D. L. (1974). Antitubularo basement membrane antibodies after renal transplantation. Transplantation, 18, 447-452.

Requests for reprints to: Dr Arthur J. Ammann, Department of Pediatrics, Immunology Section, University of California, San Francisco, California 94143, USA. 